\title{
Hybrid manufacturing of topology optimized machine tool parts through a layer laminated manufacturing method
}

\author{
Practical validation using the example of a bearing block
}

\author{
Nico Helfesrieder ${ }^{1}$ (D) Michael Neubauer ${ }^{1} \cdot$ Armin Lechler $^{1} \cdot$ Alexander Verl $^{1}$ \\ Received: 8 October 2021 / Accepted: 30 November 2021 / Published online: 13 December 2021 \\ (c) The Author(s) 2021
}

\begin{abstract}
Load-oriented lightweight structures are commonly designed based on topology optimization. For machine tool parts, they enable the reduction of moving masses and therefore increase the resource and energy efficiency of production systems. However, this usually results in complex part structures that are difficult or impossible to produce using conventional manufacturing methods. In this paper, a hybrid layer laminated manufacturing (LLM) method is proposed enabling manufacturing of topology-optimized machine tool parts. The method is referred to as hybrid, as the subtractive structuring of metal sheets is combined with the additive joining of the sheets by adhesive bonding. This enables enclosed inner cavities without support structures, which are used to approximate the optimal density distribution from a topology optimization via manufacturing. The proposed LLM method is validated on the basis of a bearing block of a ball screw feed drive. A experimental study in the time and frequency domain on a test rig confirms the principle suitability of the LLM method for the production of industrial applicable lightweight components.
\end{abstract}

Keywords Hybrid manufacturing · Layer laminated manufacturing · Topology optimization · Experimental validation

\section{Introduction}

For modern machine tools and manufacturing units, maximum performance and precision are required, as well as high resource and energy efficiency [1]. These demands cause a conflict of objectives, as material has to be saved without compromising the stiffness of the machine tools [2].

To achieve this, application-specific optimized lightweight components are crucial, besides the choice of appropriate lightweight materials [3]. Topology Optimization (TO) is an important tool for this purpose, as it is able to provide optimized material distributions within a given design space, considering different objective functions and boundary conditions [4]. A comprehensive overview of various TO approaches was given in [5] by Sigmund and Maute.

Nico Helfesrieder

nico.helfesrieder@isw.uni-stuttgart.de

Institute for Control Engineering of Machine Tools and Manufacturing Units (ISW), University of Stuttgart, Seidenstrasse 36, Stuttgart 70174, Germany
Of these, especially density-based approaches using continuous density formulations are widely used in practical applications as they can efficiently be solved by gradient-based optimization methods [5].

To ensure manufacturability, discrete $0 / 1$-solutions containing no intermediate densities $(0<\rho<1)$ are targeted. A common approach to this is the penalization of intermediate densities within the optimization routine in combination with threshold projection filters [5]. However, the solution deviates from the numerical optimum when using penalization [6]. This results in a conflict between the solution quality and the manufacturability of the numerical result.

\subsection{Manufacturing of optimized metal part structures}

Even using the aforementioned penalization and density filtering methods, very complex part structures result from TO [7]. These may be hard or even impossible to produce using conventional subtractive manufacturing processes. For 
this reason, additive manufacturing (AM) is widely used to produce such optimized part structures [7, 8].

Metal parts are of particular interest for machine tools and are vital for various applications such as joints, guides and bearings [1]. Structural optimization of metal machine tool parts helps to improve machine performance [9], energy demands [10] as well as sustainability [11], e.g. by biologically inspired stiffness designs $[12,13]$. Functionally graded lattice structures may be used to adapt TO results and provide significant stiffness enhancement compared to regular grid structures [14]. These omit the aforementioned penalization and instead mimic the numerically optimal intermediate densities by using lattices with variable thicknesses.

However, some challenges remain, such as part size limitations and cost. Especially for machine tool parts with high volumes and low per-part-cost, conventional manufacturing methods are still the preferred choice [15].

\subsection{Layer laminated manufacturing (LLM) of optimized metal parts}

The layer laminated manufacturing (LLM) is referred to as a hybrid manufacturing process as it combines additive joining of coherent layers with their subtractive structuring before or after joining. Numerous process variants in terms of the layer materials and the joining process have been subject to research within the last decades [16-20]. LLM has received limited industrial attention so far, even though it allows unique design freedoms such as enclosed inner cavities and virtually any material combination. However, the freedom of design must also be able to be used specifically, for which LLM-specific engineering tools are required. A novel slicing method that utilizes inner cavities in order to mimic intermediate densities from TO has been proposed by the authors in [21]. This allows the use of TO without penalization and permits intermediate densities to approximate the numerically optimal part structure in terms of manufacturing.

In this paper, a comprehensive approach towards the production of low-cost, structurally optimized metal parts is proposed in Sect. 2. This approach is based on previous work from [21], where a novel method for automatic modelling of optimized LLM parts was proposed and analyzed by simulation. However, practical challenges of LLM manufacturing using adhesive bonding as a joining technique are outlined in this paper and extensive tests are conducted on the resulting part properties. As a practical example to validate the proposed LLM method, the bearing block of a ball screw feed drive is topologically optimized and manufactured by bonding steel sheets. This optimized bearing block is experimentally investigated in its real assembly situation, as described in Sect. 3. Serving as reference, identical experiments are carried out using a solid bearing block as well as a bearing block, that has an almost identical geometry compared to the LLM bearing block but is manufactured using Laser Powder Bed Fusion (PBF-LB/M). The results of this comparison are shown and discussed in Sect. 4.

\section{Comprehensive LLM method}

The following section explains the proposed approach developed for modeling and producing topologically optimized metallic LLM parts.

\subsection{LLM slicing and structuring algorithm}

The basic steps along with details on implementation of the novel method for modeling topology optimized LLM components are described in [21] and illustrated schematically in Fig. 1.

First, the part to be optimized is sliced according to the build-up direction and the sheet metal thickness (1). The resulting $2 \mathrm{D}$ layers are then equipped with a virtual discretizaion called uniform manufacturing grid (UMG) 2), enabling the downstream modeling of individual cavities for each grid cell. Taking up the TO results, normalized grid cell densities are determined using piecewise linear interpolation. For each grid cell, a cavity is modeled 3 to ensure that the remaining volume is approximately equal to the normalized grid cell density. Ultimately, all individually structured layers are extruded according to the metal sheet thickness and joined together to form an assembly CAD model (4).

A bunch of manufacturing parameters is used for cavity modeling along with the size of the grid cells - for instance, the minimum producible radius or the residual bridge width between two cavities. The simulative parameter study in [21] provided the best suitable combination of manufacturing parameters for the bearing block studied. The bearing block modeled with these parameters is selected for manufacturing, which is described below.

\subsection{LLM manufacturing process}

The LLM manufacturing process is based on layer-bylayer cutting and lamination of sheets of materials. A variety of possible materials and joining methods may be combined for LLM manufacturing. Each combination provides individual chances and challenges. In this work, the adhesive bonding of metallic sheets using high-strength epoxy-based structural adhesives is examined.

First, the part sheets are manufactured subtractively, for which laser cutting is used due to the individual structuring of each sheet. Special attention needs to be paid to 


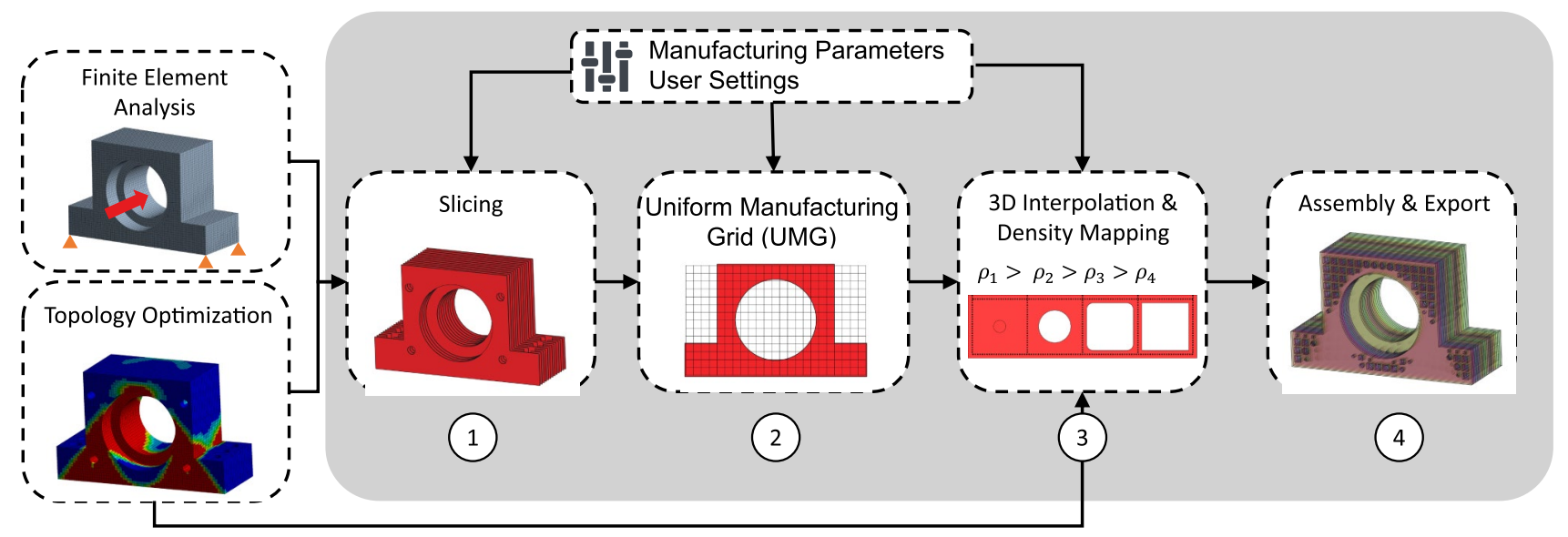

Fig. 1 Workflow of the LLM slicing and structuring method from [21] with its basic steps: Slicing; uniform manufacturing grid; density interpolation \& mapping; assembly and export

ensure low thermal warpage and clean deburring of all edges of the contact surfaces. Mechanical surface preparation is also advisable in order to set a proper surface roughness and thus improve adhesion [22]. Due to the lack of automation, the application and distribution of the adhesive is done manually. Joining the individual layers and curing them without mutual slipping or rotating is also a practical challenge at this stage. The low shear strength of the adhesive just after dispensing leads to distortion of the bonding partners even under small transverse forces. For this, depending on the part contour, mechanical frame constructions or other clamping mechanisms are to be used to secure the position. Locked against twisting, the layers are finally applied with a pressure between $0.1-12$ tons using a hydraulic press because thinner epoxy adhesive bond lines were found to provide larger bonding strengths and higher maximum shear strains [22]. Under pressure, the part is then cured in line with the curing time of the adhesive used. Once fully cured, subtractive finishing is necessary on functional surfaces and additional holes and threads are drilled.

\section{Experimental setup}

In the present work, the static and dynamic properties of the optimized LLM bearing block as well as its influence on a ball screw feed drive are investigated in detail. To demonstrate the practical applicability of the proposed LLM method, the bearing block is installed and examined on a ball screw test rig as shown in Fig. 3. The properties of the bearing block are effected by the layerwise buildup, the slicing and structuring method described in [21] as well as the adhesive films. A PBF-LB/M bearing block with almost identical geometry to the LLM bearing block is also being examined. This bearing block is basically representing the perfect adhesive layers in terms of stiffness. This way, the effects of the adhesive layers and the structuring on the loss of stiffness can be evaluated isolated from each other.

Both bearing blocks are shown in Fig. 2 and the realized level of lightweight is given in Table 1. The PBF$\mathrm{LB} / \mathrm{M}$ bearing block was printed in 1301 slices of $60 \mu \mathrm{m}$ each, which took about 45 hours. However, some internal cavities cannot be produced in the PBF-LB/M process, resulting in the weight of the PBF-LB/M bearing block being $13.4 \%$ higher compared to LLM. Furthermore, the density $\rho_{1.4404}=8.0 \mathrm{~kg} / \mathrm{dm}^{3}$ of the stainless steel used for PBF-LB/M is slightly higher than that of the structural steel $\rho_{1.0332}=7.85 \mathrm{~kg} / \mathrm{dm}^{3}$ used for LLM. In addition, the cavities visible at the contact surface on the LLM bearing block in Fig. 2 (left) are filled on the PBF-LB/M bearing block. This is due to the preprocessing of the 3D CAD file for the PBF-LB/M process, in which the cavities were filled and thickened manually with $1 \mathrm{~mm}$ of material in order to obtain a smooth surface for finishing. This visible difference between the two bearing blocks does not noticeably affect the differences in the properties of the bearing blocks, as the area fraction of the visible cavities is negligible. Since the Young's moduli of the two materials are also quite similar at
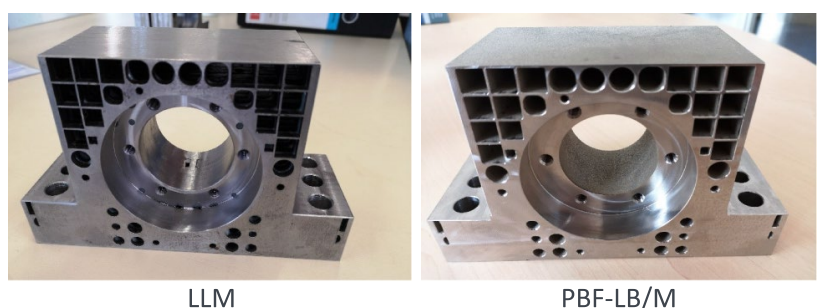

Fig. 2 Topology optimized bearing blocks manufactured through LLM and PBF-LB/M 
Table 1 Examined bearing blocks and the realized mass reduction

\begin{tabular}{lll}
\hline Part & Mass $[\mathrm{g}]$ & Mass reduction [\%] \\
\hline REF & 5100 & - \\
PBF-LB/M & 3946 & $-27,6 \%$ \\
LLM & 3480 & $-36,1 \%$ \\
\hline
\end{tabular}

Table 2 Parameters of the cascaded P-PI control used on the test rig

\begin{tabular}{ll}
\hline Parameter & $\begin{array}{l}\text { Value } \\
\text { [unit] }\end{array}$ \\
\hline position control gain $K_{\mathrm{v}}$ & $35[1 / \mathrm{s}]$ \\
velocity control proportional gain $K_{\mathrm{p}, \mathrm{n}}$ & $350[1 / \mathrm{s}]$ \\
velocity control integral gain $K_{\mathrm{i}, \mathrm{n}}$ & $50[1 / \mathrm{s}]$ \\
\hline
\end{tabular}

$E_{1.4404} \approx 200 \mathrm{kN} / \mathrm{mm}^{2}$ and $E_{1.0332} \approx 208 \mathrm{kN} / \mathrm{mm}^{2}$, comparability of the results is provided.

All experiments were performed with identical installation and boundary conditions. A cascaded P-PI control, whose parameters are shown in Table 2 is used for all measurements. To limit the influence of disturbing sources and measurement scatter, all experiments are carried several times on different days and averaged for the evaluation.

\subsection{Static force-displacement behavior}

Since the bearing block acts as a fixed bearing in the axial force transmission, its effect on the axial stiffness of the feed axis is examined. For this, the axial stiffness behavior is considered as a series connection of springs (cf. Fig. 3). The screw stiffness

$k_{\text {screw }}\left(x_{\mathrm{T}}\right)=\frac{A_{\text {screw }} E}{x_{\mathrm{T}}}$

with the cross section $A_{\text {screw }}$ and the Youngs's modulus $E$ decreases hyperbolically with the distance $x_{\mathrm{T}}$ of the machine table from the fixed bearing. Due to this, the axial stiffness of the overall mechanical system also changes when moving the machine table. To account for this influence, the experiments are performed both at the minimum $\left(x_{\mathrm{T}}=0 \mathrm{~mm}\right)$ and maximum $\left(x_{\mathrm{T}}=700 \mathrm{~mm}\right)$ position of the travel distance. Since these represent the maximum and minimum stiffness of the mechanical system respectively, no additional measurements are performed in between.

A direct drive is installed on the test rig to apply defined disturbance forces $F_{\mathrm{D}}$ to the machine table. Different constant disturbance forces are applied via this drive and the resulting displacement is determined at two locations of the mechanical system. On the one hand, the deflection of the machine table $\Delta x_{\mathrm{T}}$ is determined via the direct measuring system. On the other hand, the deflection of the bearing system $\Delta x_{\mathrm{B}}$ is determined using a laser vibrometer (resolution $0.32 \mu \mathrm{m}$ ) as shown in Fig. 3 .

Figure 4 shows the measured deflections resulting from gradually increased forces between $0-800 \mathrm{~N}$. The forces are held for $10 \mathrm{~s}$ at each step to achieve steady state. The force-deflection curves in Fig. 4 show an approximate

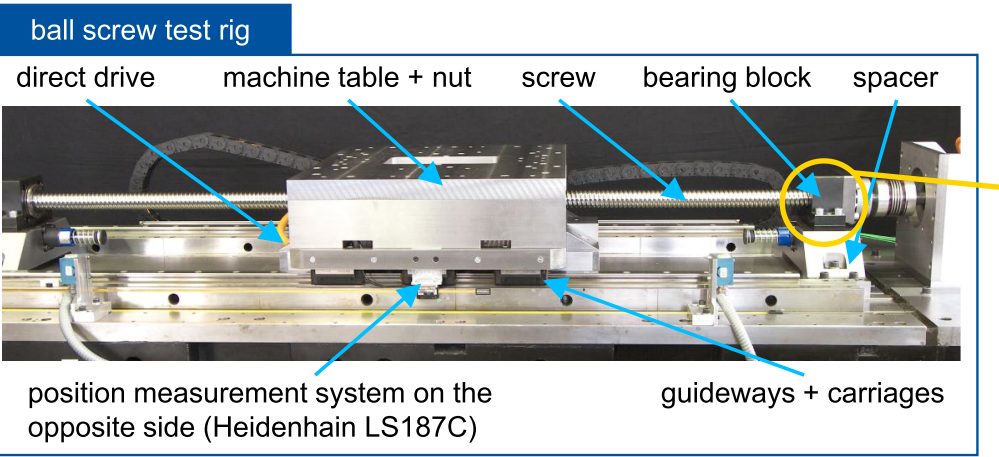

translatory equivalent mechanical model

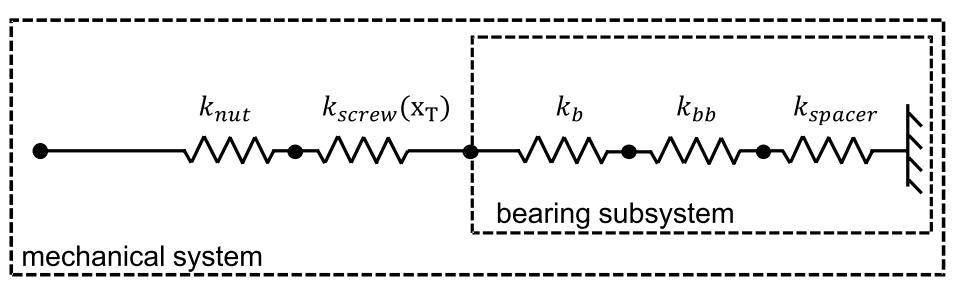

examined bearing blocks

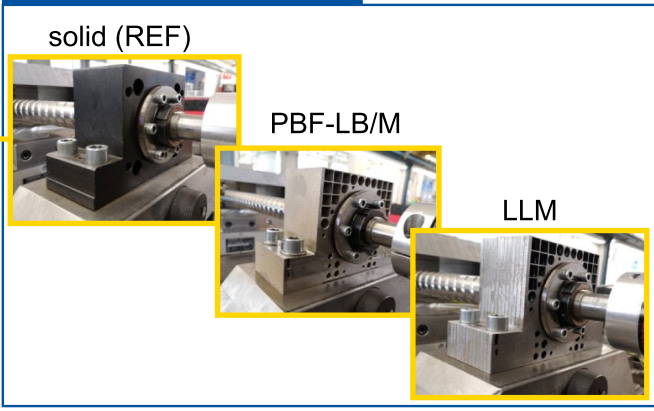

measurement on the bearing subsystem

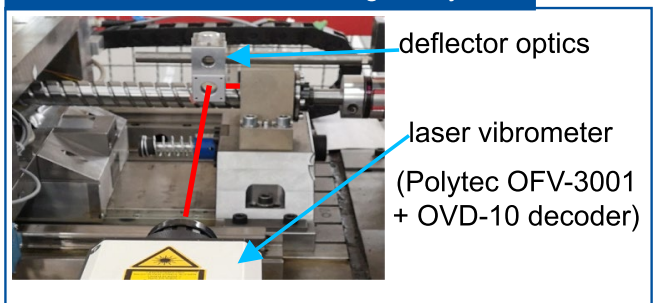

Fig. 3 Experimental setup on the ball screw test rig including examined bearing blocks in mounted state (upper right) as well as a translatory equivalent mechanical model (lower left) 
linear relationship within the investigated force range as well as a superimposed hysteresis behavior, both for the bearing subsystem and for the overall mechanical system. This is in line with the expectations, as the forces are in the range of typical axial operating loads of a ball screw feed drive. The hysteresis may be explained by mechanical settling effects due to relative motion in the bearings and bolted connections. Linear regression is used to calculate the balancing lines shown, whose gradients in each case corresponds to the static stiffness.

\subsection{Mechanical frequency response}

Changing the static and dynamic properties of the bearing block not only affects the static stiffness, but also the frequency-dependent transmission behavior of the feed drive axis. A common method for assessing this behavior is the mechanical frequency response $G_{\text {Mech }}=\dot{x}_{\mathrm{T}} / \dot{x}_{\mathrm{M}}$, which relates the motion of the machine table $\dot{x}_{\mathrm{T}}$ to the motor velocity $\dot{x}_{\mathrm{M}}=\dot{\Theta}_{\mathrm{M}} i_{\mathrm{bs}}$ with the ball screw transmission ratio $i_{\mathrm{bs}}$. A logarithmic sine sweep, whose parameters are shown in Table 3, is used as excitation signal for the machine table velocity $\dot{x}_{\mathrm{T}}$. A speed control is used here instead of a position control, see Table 2.

As the axial screw stiffness decreases with increasing travel distance, the measurements are performed at the minimum and maximum possible start position $x_{\mathrm{T}, \mathrm{Star}}$, which is
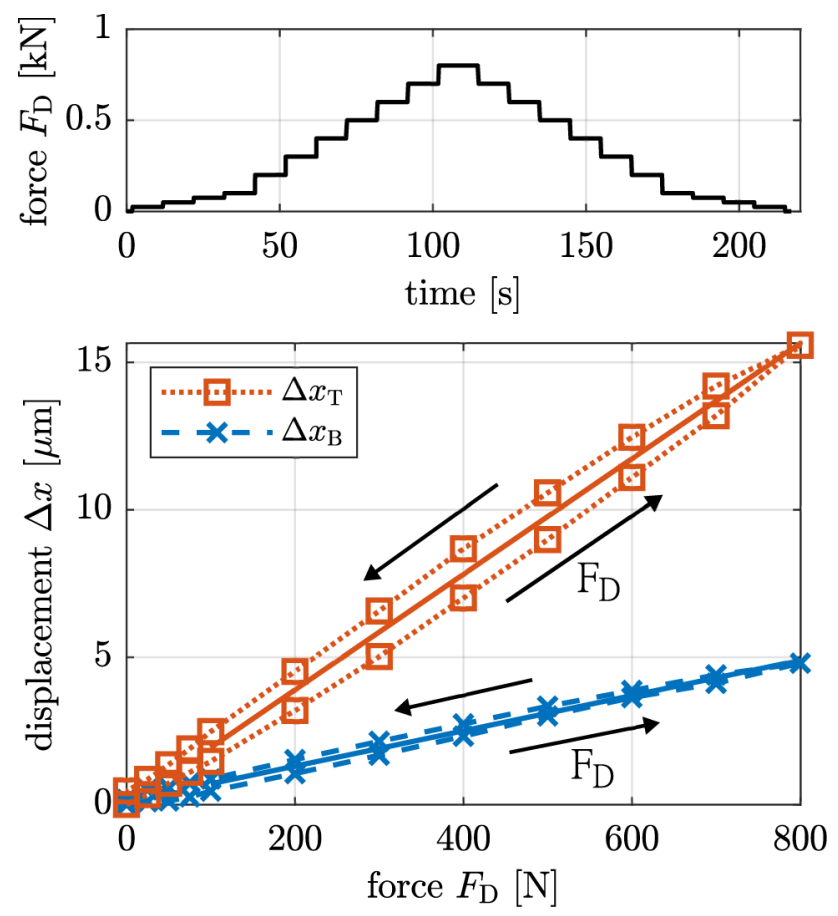

Fig. 4 Load profile (upper) used for stiffness measurement and the resulting force-displacement curves (lower) at the machine table and at the bearing subsystem under use of the PBF-LB/M bearing block determined by the offset velocity $\dot{x}_{\mathrm{T}, \mathrm{Offset}}$ and the resulting travel distance.

\subsection{Compliance frequency response}

Another measure for evaluation of the dynamic behavior is the compliance frequency response. It describes the elastic, frequency-dependent deflection resistance under a dynamic disturbance force acting on the machine table, such as during a milling process. Machining limits depend to a large extent on the dynamic behavior, which in turn results from the stiffness, structural mass as well as damping [23]. The stiffness is expected to be compromised by the adhesive layers of the LLM bearing block, as well as the material removed. Using the direct drive, a dynamic disturbance force $F_{\mathrm{D}}$ is applied to the machine table as a sweep with the values shown in Table 4 . The relation of the resulting machine table deflection $\Delta x_{\mathrm{T}}$ to the disturbance force $F_{\mathrm{D}}$ results in the transfer function $G_{\text {Comp }}=\Delta x_{\mathrm{T}} / F_{\mathrm{D}}$.

Experiment variations result from the position $x_{\mathrm{T}}$ of the machine table as well as the cascaded P-PI control being active or inactive respectively. Inactive control means that the motor is not powered, but is blocked by a mechanical brake. With active control, the motor reacts to the disturbance forces and compensates for the positioning errors measured on the position measuring system, which results in a different transmission behavior.

\subsection{Damping behavior}

Damping is a key factor for improving machine performance, as the induced energy losses result in less sensitivity to vibration [23]. While the adhesive layers presumably reduce the stiffness of LLM parts, the damping can be expected to increase. It has already been found that hybrid

Table 3 Parameters used for measurement of the mechanical frequency response

\begin{tabular}{ll}
\hline Parameter & Value [unit] \\
\hline Amplitude & $0.5[\mathrm{~mm} / \mathrm{s}]$ \\
Frequency range & $1-350[\mathrm{~Hz}]$ \\
Offset velocity $\dot{x}_{\mathrm{t}, \text { Offset }}$ & $8[\mathrm{~mm} / \mathrm{s}]$ \\
\hline
\end{tabular}

Table 4 Parameters used for measurement of the compliance frequency response

\begin{tabular}{ll}
\hline Parameter & Value [unit] \\
\hline Amplitude & $300[\mathrm{~N}]$ \\
Frequency range & $0.1-150[\mathrm{~Hz}]$ \\
Offset velocity $\dot{x}_{\mathrm{t}, \text { Offset }}$ & $0[\mathrm{~mm} / \mathrm{s}]$ \\
\hline
\end{tabular}


machine elements lead to considerable increases in part damping by combining steel and composites [24-26]. However, the damping of machines is mainly dominated by the motor and friction effects in connection interfaces [1, 23].

The extent to which increased part damping has a positive effect on the damping of the overall system and thus on the dynamic properties of the ball screw test rig is investigated. The decay behavior in the time domain is investigated for this purpose. A step excitation force of $F_{\mathrm{D}}=800 \mathrm{~N}$ is applied using the direct drive.

The deflections at the machine table $\Delta x_{\mathrm{T}}$ are measured for minimum and maximum machine table position, as shown in Fig. 5. A clear oscillation with obvious damping is present in all cases. The level of deflection is slightly higher for LLM compared to the other bearing blocks, which indicates a reduction in stiffness. Also for the maximum table position $x_{\mathrm{T}}=700 \mathrm{~mm}$, there is a higher deflection of the machine table because of lower screw stiffness.

An exemplary decay curve is shown in Fig. 6 to demonstrate the damping identification. The position signal of the machine table is numerically differentiated to compensate for drift effects due to sensor drift and hysteresis behavior of the feed drive. Starting at the second peak of the decay curve, intervals of equal size are analyzed for each run. The damping of the observed vibration results from the ratio of subsequent peaks expressed as the decay rate of an exponential function, which is fitted to the upper envelope of the decay curve and is called e-Fit in the following [27].

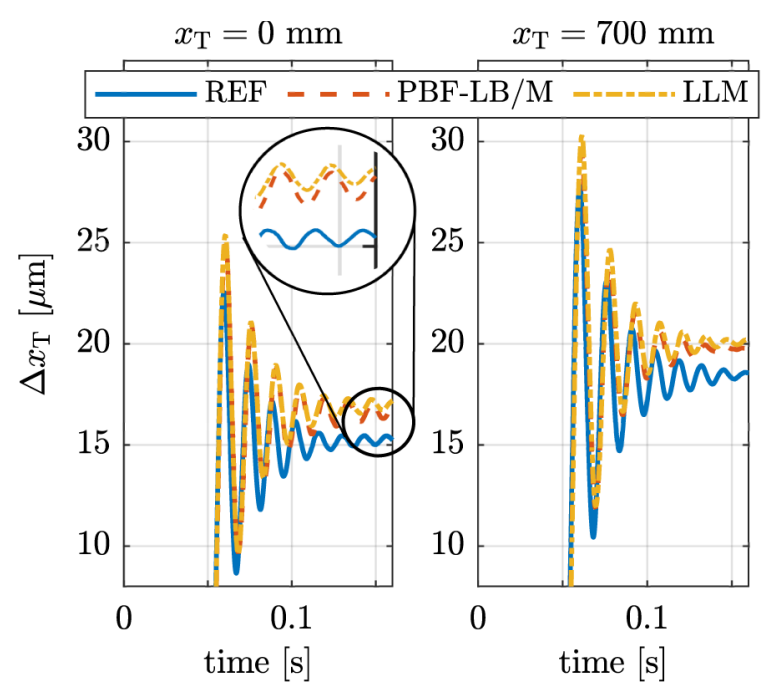

Fig. 5 Exemplary decay curves used for damping identification on the overall mechanical system for minimum (left) and maximum (right) machine table position

\section{Results and discussion}

In the following, the main results are shown and discussed, using the same pattern as in Sect. 3 .

\subsection{Static force-displacement behavior}

The stiffness values derived in this way for the examined bearing blocks are visualized in Fig. 7, in each case using the mean value and the variation range. A significant reduction in the stiffness of the bearing subsystem by $20-22 \%$ using the LLM bearing block is obtained, while the PBF-LB/M and the solid bearing block show almost identical mean values and overlapping variation widths. For the overall system, the influence is less clear and also less significant with 6-9\% stiffnes reduction using the LLM bearing block. This is explained by the fact that the axial stiffness $k_{\text {screw }}$ of the screw has the lowest value in the overall system and is therefore dominant as the weakest spring (cf. Fig. 3).

\subsection{Mechanical frequency response}

The mechanical frequency responses for table start position $x_{\mathrm{T}, \mathrm{Start}}=0 \mathrm{~mm}$, as shown in Fig. 8 top, indicate a qualitatively similar behavior for all bearing blocks investigated. The first mechanical eigenfrequency is reduced by about $6.2 \%$ to $57.40 \mathrm{~Hz}$ using the LLM bearing block compared to the PBF-LB/M and the solid bearing block, which are almost identical at $60.69 \mathrm{~Hz}$ and $61.18 \mathrm{~Hz}$ respectively. This decrease in eigenfrequency is explained by the reduced axial stiffness of the system with LLM bearing block as described in Sect. 4.1. For the maximum possible start position $x_{\mathrm{T}, \text { Start }}=389.7 \mathrm{~mm}$, the eigenfrequencies are slightly lower. The solid bearing block and the PBF-LB/M again

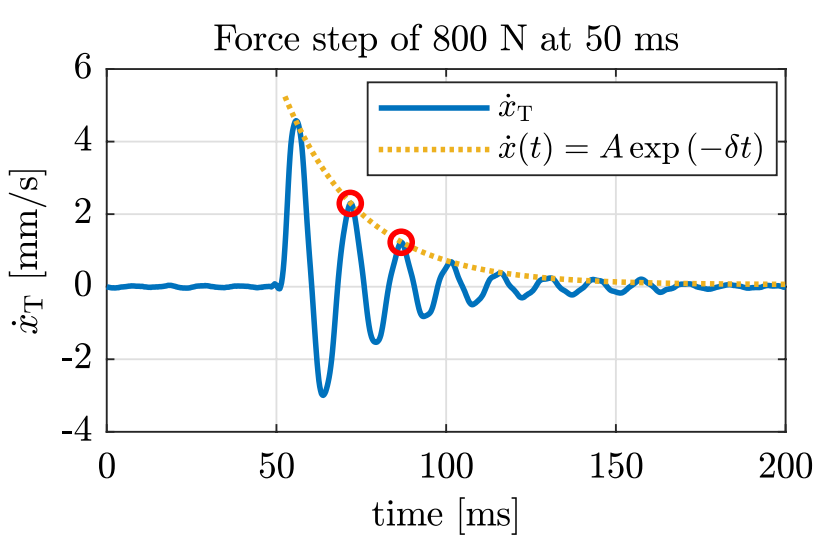

Fig. 6 Exemplary damping ratio calculation using the decay curves of the table speed 

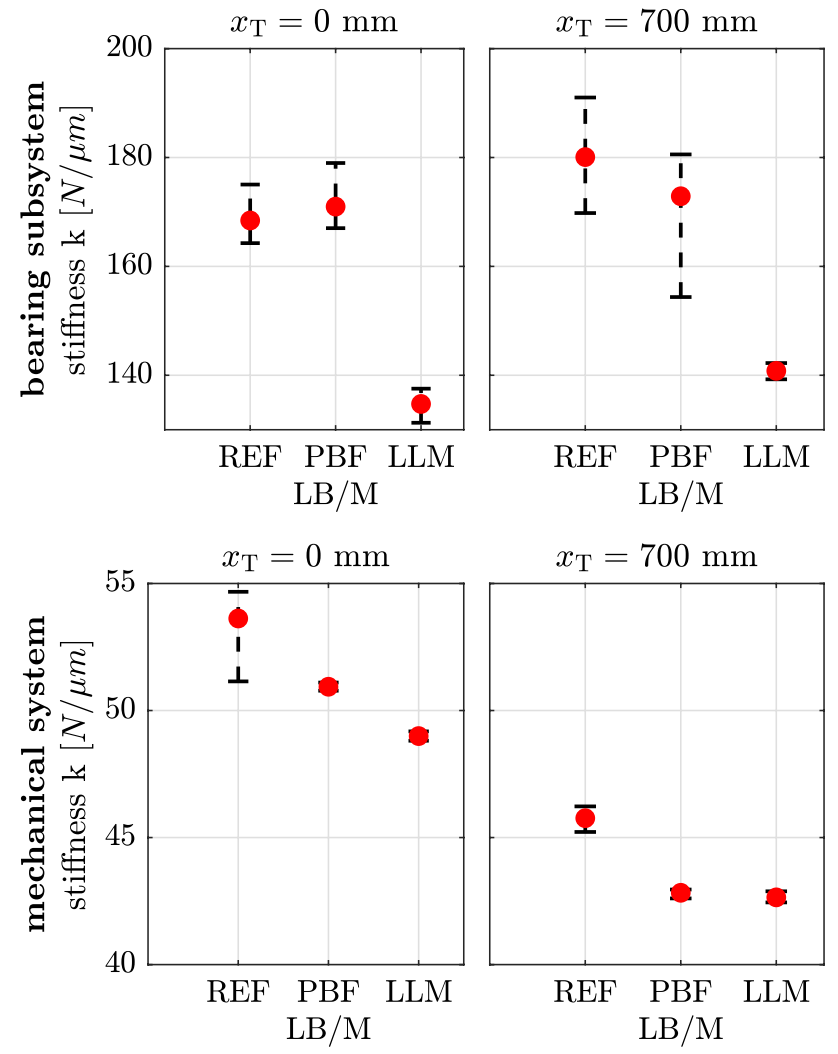

Fig. 7 Static stiffness for bearing subsystem (upper) and complete mechanical system (lower) obtained through linear regression from static force-displacement curves

indicate almost identical values with 58.85 and $59.10 \mathrm{~Hz}$, while the LLM is about $3.6 \%$ lower at $56.66 \mathrm{~Hz}$.

The comparison between PBF-LB/M and LLM leads to the conclusion that it is not the topology but the adhesive layers that are mainly responsible for the reduced stiffness and the reduced eigenfrequency. This supports the functionality of the slicing method described in Sect. 2, but also shows that LLM fabrication is so far only viable for applications with sufficient stiffness margins.

\subsection{Compliance frequency response}

The measured compliance frequency responses are shown in Fig. 9 for minimum and maximum table position as well as active and inactive P-PI control as described in Sect. 3.3.

The maximum dynamic compliance is considered, which is indicated by the peaks of the curves. For all studied variants, the highest peak compliance is observed when using the LLM bearing block, which is consistent with the results from Sects. 4.1 and 4.2. For inactive control, this peak compliance is $7.1-7.7 \%$ higher than for the solid bearing block, and for active control it is between 9.9 and $20.6 \%$ higher depending on the table position $x_{\mathrm{T}}$. For the PBF-LB/M
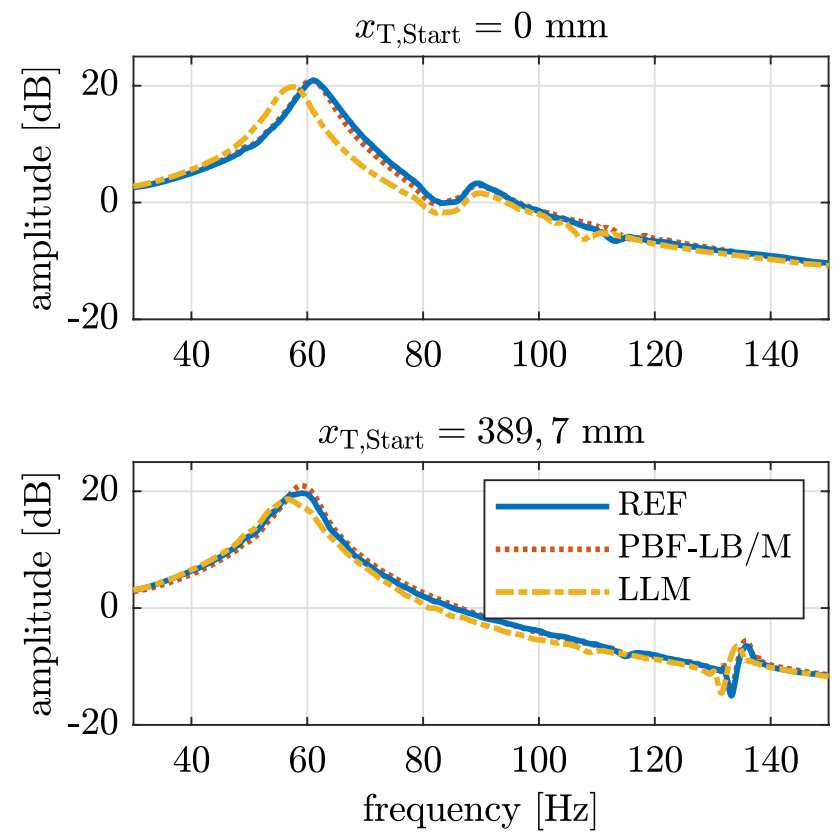

Fig. 8 Mechanical frequency responses of the ball screw drive with variation of the bearing blocks

bearing block, the difference is about $6.2-6.3 \%$ for inactive control and $2.6-10.1 \%$ for active control.

However, no consistent distance between the peak compliance of the bearing blocks is observed. Especially for the maximum table position, there is an increased measurement scatter and, with active control, significantly larger differences between LLM and PBF-LB/M than for inactive control. Reasons for this could be the friction behavior in the rear area due to surface rust on the screw as well as not ideally aligned guide carriages, since the software limit switch is located shortly behind this position.

Furthermore, the peak frequencies for LLM and PBF$\mathrm{LB} / \mathrm{M}$ are about $1.7-4.0 \%$ lower than those of the solid bearing block. This decrease is on the one hand due to the reduced stiffness in axial direction and on the other hand due to static friction as no offset velocity is used.

\subsection{Damping behavior}

The damping rates are determined via an e-Fit according to Sect. 3.4 and are shown with mean value and scatter width in Fig. 10.

The damping with LLM is the highest at both table positions and is $3.9 \%\left(x_{\mathrm{T}}=0 \mathrm{~mm}\right)$ and $14.0 \%\left(x_{\mathrm{T}}=700 \mathrm{~mm}\right)$ higher than with the solid bearing block. There is also an increase in damping of 0.6 and $10.7 \%$ using the PBF-LB/M bearing block.

Particularly noticeable are the differences in damping for both table positions with the PBF-LB/M bearing block, 

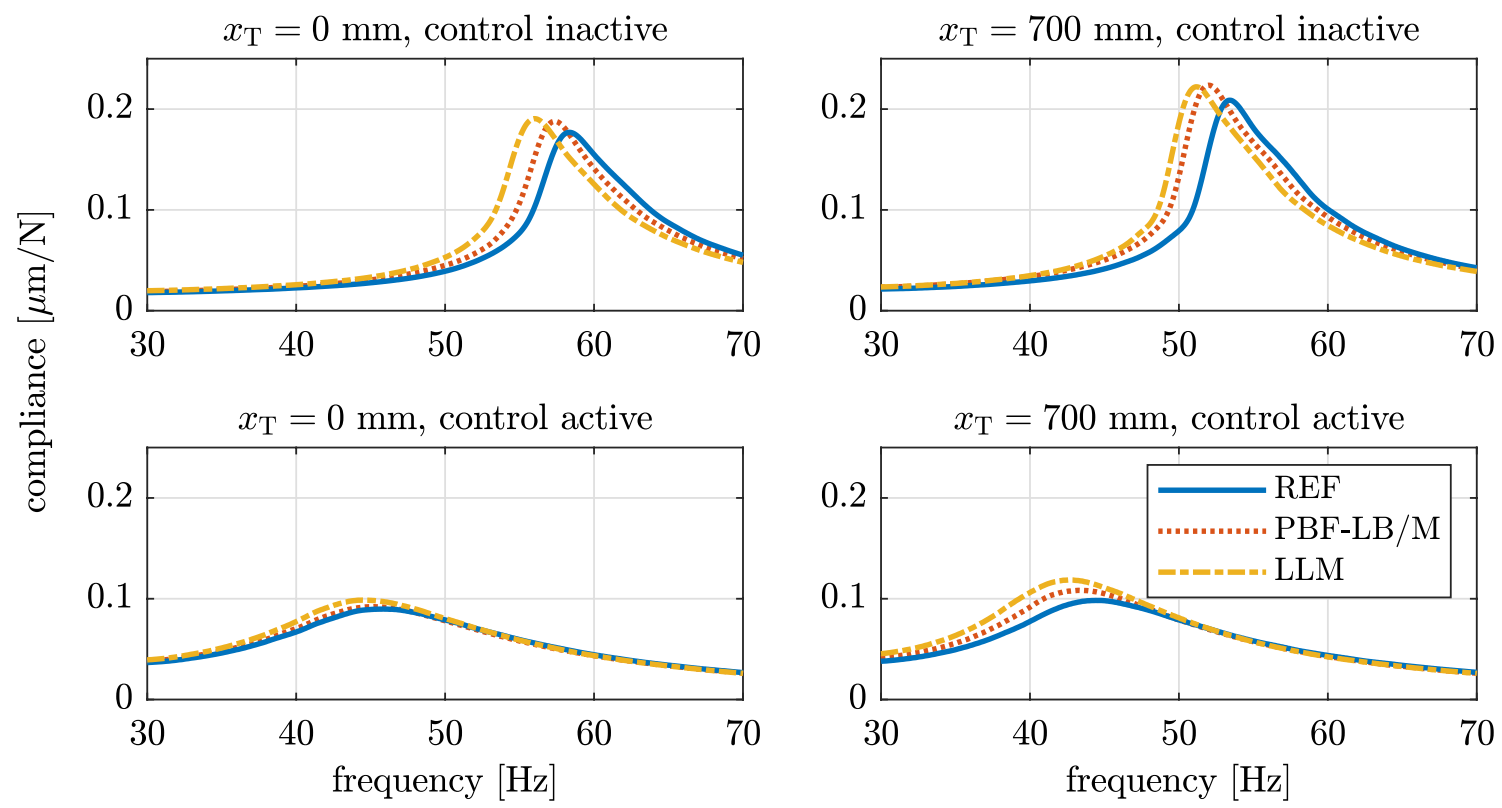

Fig. 9 Compliance frequency responses of the ball screw drive with variation of the bearing block used, the table position as well as active/inactive control
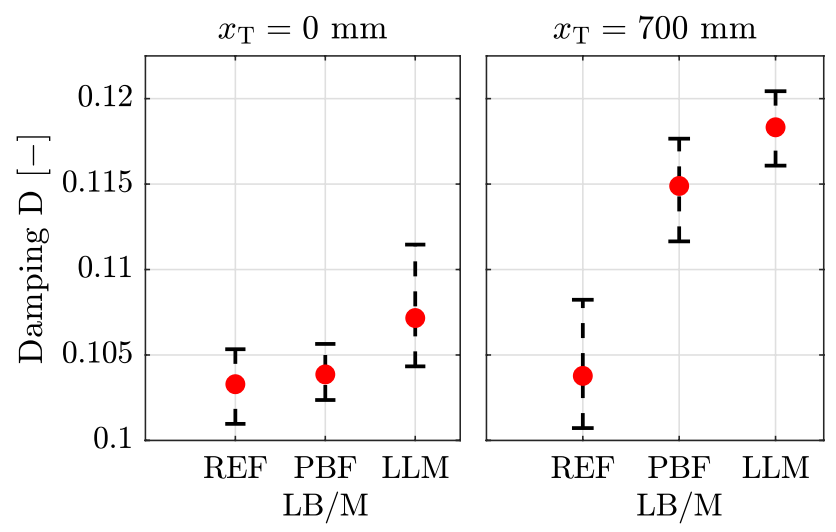

Fig. 10 Damping rates mean value (red dot) and scatter width for the mechanical system, averaged over four runs at front $\left(x_{\mathrm{T}}=0 \mathrm{~mm}\right)$ and rear $\left(x_{\mathrm{T}}=700 \mathrm{~mm}\right)$ table position

which cannot be readily explained physically. Moreover, other components, such as friction or the motor control, essentially contribute to the damping of the overall system. Nevertheless, it can be stated that the damping is increased using the bonded LLM bearing block, despite the higher scatter of the measurements with the LLM bearing block. The increased damping could be used specifically, for example, to passively damp machine elements or axes that are very sensitive to vibration.

\section{Conclusion}

In this paper, a novel method for hybrid manufacturing of topology optimized metal parts, a LLM method, was experimentally analyzed in detail. The inner optimization through enclosed cavities is a unique feature of this method and is able to provide industrially applicable lightweight parts for machine tools. The bearing block of a ball screw test rig served as a real-world example for the evaluation of the method. Its mass was reduced by $36.1 \%$ using LLM whereby PBF-LB/M could only achieve $27.6 \%$. However, the investigations of the static and dynamic stiffness also showed that both the structuring of the individual layers and the adhesive layers lead to a reduction in stiffness, which must be considered. For parts with stiffness margins, the presented method can nevertheless be applied well without compromising the properties of the machine or axis too much. Another effect shown was an increase in damping, although damping results essentially from motor control and friction. However, this effect might be used to selectively introduce passive damping in low-friction, vibration-prone axes to improve their dynamic behavior.

The next possible research steps include an investigation of the correlation between the LLM process parameters and the resulting component properties. Based on the enhanced process knowledge, the LLM process should also be further automated to ensure consistent quality and to minimize waste. Potential practical applications for the combination of steel sheets and structural adhesive studied in this paper are low-cost lightweight components and passive damped 
machine tool parts. Moreover, in addition to the bearing block, another machine tool part whose dynamic behavior has a greater influence on the overall machine behavior (e.g. the machine table) should be used for validation purposes. Further variants of the LLM process should also be considered for other applications in the future. For example, alternative joining methods such as ultrasonic welding or soldering may be used to improve the stiffness of the LLM components.

Acknowledgements This work is supported by the German Research Foundation (DFG) under project number 246665445. The authors gratefully acknowledge the support by DFG. The authors would also like to thank Steffen Trefz for his support during the experiments in the context of his master thesis.

Funding Open Access funding enabled and organized by Projekt DEAL.

\section{Declarations}

Conflict of interest The authors declare that they have no conflict of interest.

Open Access This article is licensed under a Creative Commons Attribution 4.0 International License, which permits use, sharing, adaptation, distribution and reproduction in any medium or format, as long as you give appropriate credit to the original author(s) and the source, provide a link to the Creative Commons licence, and indicate if changes were made. The images or other third party material in this article are included in the article's Creative Commons licence, unless indicated otherwise in a credit line to the material. If material is not included in the article's Creative Commons licence and your intended use is not permitted by statutory regulation or exceeds the permitted use, you will need to obtain permission directly from the copyright holder. To view a copy of this licence, visit http://creativecommons.org/licenses/by/4.0/.

\section{References}

1. Möhring HC, Brecher C, Abele E, Fleischer J, Bleicher F (2015) Materials in machine tool structures. CIRP Ann 64(2):725-748. https://doi.org/10.1016/j.cirp.2015.05.005

2. Kroll L, Blau P, Wabner M, Frieß U, Eulitz J, Klärner M (2011) Lightweight components for energy-efficient machine tools. CIRP J Manuf Sci Technol 4(2):148-160. https://doi.org/10.1016/j.cirpj. 2011.04.002

3. Möhring HC, Müller M, Krieger J, Multhoff J, Plagge C, de Wit J, Misch S (2020) Intelligent lightweight structures for hybrid machine tools. Prod Eng 14(5-6):583-600. https://doi.org/10. 1007/s11740-020-00988-3

4. Bendsøe MP, Sigmund O (2011) Topology optimization: theory, methods, and applications, 2nd edn, corrected printing edn. Engineering online library, Springer, Berlin. http://swbplus.bsz-bw.de/ bsz109502531 cov.htm

5. Sigmund O, Maute K (2013) Topology optimization approaches. Struct Multidiscip Optim 48(6):1031-1055. https://doi.org/10. 1007/s00158-013-0978-6
6. Dadalau A, Hafla A, Verl A (2009) A new adaptive penalization scheme for topology optimization. Prod Eng 3(4-5):427-434. https://doi.org/10.1007/s11740-009-0187-8

7. Plocher J, Panesar A (2019) Review on design and structural optimisation in additive manufacturing: towards next-generation lightweight structures. Mater Des 183:108164. https://doi.org/ 10.1016/j.matdes.2019.108164

8. Bandyopadhyay A, Traxel KD (2018) Invited review article: metal-additive manufacturing-modeling strategies for application-optimized designs. Addit Manuf 22:758-774. https://doi. org/10.1016/j.addma.2018.06.024

9. Fleischer J, Munzinger C, Tröndle M (2008) Simulation and optimization of complete mechanical behaviour of machine tools. Prod Eng 2(1):85-90. https://doi.org/10.1007/ s11740-007-0069-X

10. Ji Q, Li C, Zhu D, Jin Y, Lv Y, He J (2020) Structural design optimization of moving component in cnc machine tool for energy saving. J Clean Prod 246:118976. https://doi.org/10.1016/j.jclep ro.2019.118976

11. Herrmann C, Dewulf W, Hauschild M, Kaluza A, Kara S, Skerlos S (2018) Life cycle engineering of lightweight structures. CIRP Ann 67(2):651-672. https://doi.org/10.1016/j.cirp.2018.05.008

12. Li B, Hong J, Liu Z (2014) Stiffness design of machine tool structures by a biologically inspired topology optimization method. Int J Mach Tools Manuf 84:33-44. https://doi.org/10.1016/j.ijmac htools.2014.03.005

13. Zhang H, Ding X, Dong X, Xiong M (2018) Optimal topology design of internal stiffeners for machine pedestal structures using biological branching phenomena. Struct Multidiscip Optim 57(6):2323-2338. https://doi.org/10.1007/s00158-017-1862-6

14. Panesar A, Abdi M, Hickman D, Ashcroft I (2018) Strategies for functionally graded lattice structures derived using topology optimisation for additive manufacturing. Addit Manuf 19:81-94. https://doi.org/10.1016/j.addma.2017.11.008

15. Vafadar A, Guzzomi F, Rassau A, Hayward K (2021) Advances in metal additive manufacturing: a review of common processes, industrial applications, and current challenges. Appl Sci 11(3):1213. https://doi.org/10.3390/app11031213

16. Gibson I, Rosen D, Stucker B (2015) Sheet lamination processes. In: Gibson I, Rosen D, Stucker B (eds) Additive manufacturing technologies. Springer. New York, pp 219-244. https://doi. org/10.1007/978-1-4939-2113-3_9

17. Obikawa T, Yoshino M, Shinozuka J (1999) Sheet steel lamination for rapid manufacturing. J Mater Process Technol 89-90:171-176. https://doi.org/10.1016/S0924-0136(99)00027-8

18. Levy A, Miriyev A, Sridharan N, Han T, Tuval E, Babu SS, Dapino MJ, Frage N (2018) Ultrasonic additive manufacturing of steel: Method, post-processing treatments and properties. J Mater Process Technol 256:183-189. https://doi.org/10.1016/j. jmatprotec.2018.02.001, http://www.sciencedirect.com/science/ article/pii/S0924013618300463

19. Modaresahmadi S, Barnett D, Baninajar H, Bird JZ, Williams WB (2021) Structural modeling and validation of laminated stacks in magnetic gearing applications. Int J Mech Sci 192:106133. https:// doi.org/10.1016/j.ijmecsci.2020.106133

20. Yamasaki K, Fukuda S, Murakami T, Maekawa K (2007) Fabrication of functionally porous structures by the sheet lamination method. Mater Sci Forum 561-565:1711-1714. https://doi.org/ 10.4028/www.scientific.net/MSF.561-565.1711

21. Helfesrieder N, Lechler A, Verl A (2020) Method for generating manufacturable, topology-optimized parts for laminated layer manufacturing. Procedia CIRP 93:38-43. https://doi.org/10. 1016/j.procir.2020.04.048, http://www.sciencedirect.com/scien ce/article/pii/S2212827120306144

22. Zhang D, Huang Y (2021) Influence of surface roughness and bondline thickness on the bonding performance of epoxy adhesive 
joints on mild steel substrates. Prog Org Coat. https://doi.org/10. 1016/j.porgcoat.2021.106135

23. Kulíšek V, Kolar P, Vrba P, Smolík J, Janota M, Růžička M, Machálka M, (2021) On passive damping in machine tool hybrid structural parts. Int J Adv Manuf Technol. https://doi.org/10.1007/ s00170-021-06865-2

24. Sarlin E, Liu Y, Vippola M, Zogg M, Ermanni P, Vuorinen J, Lepistö T (2012) Vibration damping properties of steel/rubber/ composite hybrid structures. Compos Struct 94(11):3327-3335. https://doi.org/10.1016/j.compstruct.2012.04.035

25. Wang X, Li X, Yu RP, Ren JW, Zhang QC, Zhao ZY, Ni CY, Han B, Lu TJ (2020) Enhanced vibration and damping characteristics of novel corrugated sandwich panels with polyurea-metal laminate face sheets. Compos Struct 251:112591. https://doi.org/10.1016/j. compstruct.2020.112591, http://www.sciencedirect.com/science/ article/pii/S0263822320309582
26. Wang X, Li X, Yue ZS, Yu RP, Zhang QC, Du SF, Yang ZK, Han B, Lu TJ (2021) Optimal design of metallic corrugated sandwich panels with polyurea-metal laminate face sheets for simultaneous vibration attenuation and structural stiffness. Compos Struct 256:112994. https://doi.org/10.1016/j.compstruct.2020.112994

27. Jacobson KE, Kiviaho JF, Kennedy GJ, Smith MJ (2019) Evaluation of time-domain damping identification methods for flutterconstrained optimization. J Fluids Struct 87:174-188. https://doi. org/10.1016/j.jfluidstructs.2019.03.011

Publisher's Note Springer Nature remains neutral with regard to jurisdictional claims in published maps and institutional affiliations. 